\title{
Efecto de la fragmentación forestal sobre la estructura vegetacional de las poblaciones amenazadas de Legrandia concinna (Myrtaceae) del centro-sur de Chile
}

\author{
Effect of forest fragmentation on vegetation structure of Legrandia concinna (Myrtaceae) \\ threatened populations in south-central Chile
}

ADISON ALTAMIRANO*, CRISTIAN ECHEVERRÍA \& ANTONIO LARA

Instituto de Silvicultura, Facultad de Ciencias Forestales, Universidad Austral de Chile, Casilla 567, Valdivia, Chile

*e-mail para correspondencia: aaltamir@uach.cl

\begin{abstract}
RESUMEN
La fragmentación del hábitat es una de las principales amenazas para la integridad de los ecosistemas. En este estudio se evaluó el efecto de la fragmentación sobre la estructura de la vegetación en poblaciones de la especie arbórea Legrandia concinna en el centro-sur de Chile. Se describió el patrón de ocupación de las poblaciones de Legrandia en fragmentos remanentes de bosques nativos y se evaluaron las relaciones entre índices de paisaje y variables de estructura de la vegetación. Los índices de paisaje (área, índice de proximidad media, forma y distancia al borde de fragmentos) fueron aplicados a mapas de cobertura forestal generados a partir de una imagen satelital Landsat ETM+ del año 2001. Los resultados mostraron una alta predominancia de fragmentos de reducido tamaño, altos niveles de aislamiento y formas regulares. El patrón de ocupación de las poblaciones de Legrandia sugiere que las poblaciones ocupan preferentemente fragmentos de menor tamaño y mayor aislamiento. No hubo diferencias significativas entre la distribución de frecuencias de los fragmentos con presencia y sin presencia de Legrandia en cuanto a área y aislamiento. Sin embargo, se obtuvo que el tamaño, aislamiento y la distancia al borde del fragmento se relacionara significativamente con la presencia de la especie. Como medida de conservación de la especie, se recomienda incluir dentro de áreas protegidas a aquellas poblaciones con mayor riesgo a desaparecer, promover su conservación ex situ y establecer un programa de monitoreo. El estudio sienta un precedente respecto a la vinculación entre información a escala de paisaje proveniente de imágenes satelitales e información de estructura de la vegetación medida en terreno, para evaluar el estado de conservación de poblaciones de especies amenazadas que cubren extensiones restringidas de territorio. Esta innovación metodológica ofrece la posibilidad de monitorear los cambios producidos en el tiempo a fin de evaluar la efectividad de determinadas estrategias de conservación para estas poblaciones.
\end{abstract}

Palabras clave: Legrandia concinna, fragmentación, índices de paisaje, conservación.

\begin{abstract}
Habitat fragmentation is one of the main threats to ecosystems integrity. In this study we assessed the influence of forest fragmentation on vegetation structure of populations of tree specie Legrandia concinna in south-central Chile. Occupancy pattern of Legrandia populations in remaining fragments of native forests was described, and relationship between landscape indices and vegetation structure variables were evaluated. Landscape indices, such as area, proximity mean index, shape and edge distance to fragment were applied to forest cover maps generated from a Landsat ETM+ satellite scene for the year 2001. Results showed high predominance of reduced size fragments, high isolation levels and regulars shapes of the fragments. Occupancy pattern suggests that Legrandia populations are found mainly in small and more isolated fragments. No significant difference was found between fragment distribution with and without presence of Legrandia related to size and isolation. However, we found that size, isolation and distance to fragment edge are significantly related to the presence of this specie. As conservation measures for this specie, we propose to include populations with high disappearance risk in protected areas, promote ex situ conservation and set up a monitoring program. This study sets a precedent related by linking landscape scale information obtained from satellite imagery and vegetation structure information measured in the field with conservation status of threatened populations. This innovative method allows monitoring changes over time to assess effectiveness of conservation strategies for these populations.
\end{abstract}

Key words: Legrandia concinna, fragmentation, landscape indices, conservation. 


\section{INTRODUCCIÓN}

La fragmentación del hábitat ha sido reconocida como una de las principales amenazas para los ecosistemas (Dale \& Pearson 1997, Noss 2000, Armenteras et al. 2003), manifestando sus principales efectos a través de la reducción de la biodiversidad y aumentando el aislamiento de hábitats (Skole \& Tuker 1993). Este proceso también puede tener efectos negativos sobre poblaciones amenazadas modificando la dinámica de las especies, lo cual puede conducir a alterar la persistencia de las poblaciones en el tiempo (Tomimatsu \& Ohara 2003). En Chile, estudios realizados en bosques de ruil muestran los efectos negativos de la fragmentación sobre estos ecosistemas como el aumento del aislamiento y pérdida de conectividad entre los fragmentos (Bustamante \& Grez 1995, Bustamante \& Castor 1998, Grez et al. 1998). En el centro y sur de Chile, se ha reportado el efecto negativo de la fragmentación forestal sobre la diversidad y abundancia de aves (Willson et al. 1994, Estades \& Temple 1999, Vergara \& Simonetti 2004), así como en la calidad de semillas de árboles (Henríquez 2004).

La fragmentación de un hábitat puede medirse cuantificando cambios en la estructura espacial del paisaje, lo cual se refiere a la relación espacial entre parches o fragmentos (Turner et al. 2001). Estas mediciones son realizadas a través de métricas espaciales o índices de paisaje, y su uso resulta de gran utilidad, ya que pueden proveer información acerca de la ocurrencia de procesos de deforestación y fragmentación (Franklin 2001, Lausch \& Hertzog 2002, Fitzsimmons 2003, Li et al. 2004). Estos índices de paisaje pueden ser aplicados a mapas temáticos, los cuales pueden ser generados a partir de imágenes satelitales (Kerr \& Ostrovsky 2003).

Los cambios producidos por la fragmentación se ven reflejados en la estructura espacial del paisaje como el tamaño, forma o posición de los fragmentos en el paisaje (Turner et al. 2001). Algunos estudios que han aplicado índices de paisaje como el tamaño de fragmentos sugieren que altos niveles de fragmentación están asociados a predominancia de fragmentos de menores tamaños (Gigord et al. 1999, Armenteras et al. 2003, Fitzsimmons 2003, Cayuela et al. 2006). Otros índices como aislamiento y forma de fragmentos también han sido aplicados para evaluar el grado de fragmentación de ecosistemas, reportándose mayores niveles de fragmentación en áreas dominadas por fragmentos con mayor grado de aislamiento y formas regulares (Bustamante \& Castor 1998, Echeverría et al. 2006). Dichos cambios, a su vez, pueden modificar ciertos atributos ecológicos de los hábitat afectados por fragmentación (Saunders et al. 1991). Es así como la fragmentación puede afectar los procesos de polinización y producción de frutos (Gigord et al. 1999). También pueden manifestarse efectos demográficos y genéticos, los cuales contribuyen a disminuir la interacción de las plantas con sus polinizadores, producen cambios microclimáticos y reducen la heterocigocidad, afectando la viabilidad de una población a futuro (Tomimatsu \& Ohara 2003). Estudios de otras regiones han analizado la respuesta a la fragmentación de especies animales mediante análisis del patrón de ocupación de los fragmentos con y sin presencia de la especie, lo cual ha permitido relacionar la ocurrencia de la especie con índices de paisaje como tamaño y aislamiento de fragmentos (Verboom \& van Apeldoorn 1990, Virgós \& García 2002, Banks et al. 2005).

Debido a los altos niveles de endemismo de los bosques templados de Chile (Armesto et al. 1998), estos ecosistemas han sido seleccionados por la iniciativa Global 200 de la WWF y el Banco Mundial como una de las ecorregiones más prioritarias para la conservación a nivel mundial (Dinerstein et al. 1995). Dentro de esta zona se distribuye Legrandia concinna (Phil.) Kausel, especie que actualmente se encuentra catalogada como "Vulnerable", de acuerdo con el "Libro Rojo de la Flora Terrestre de Chile" (Benoit 1989) y como "Rara" de acuerdo a "IUCN 1997 Red List of Threatened Plants" (Walter \& Gillett 1998). En un estudio reciente, basado en criterios actualizados de la UICN, Legrandia fue incluida en la categoría "En Peligro Crítico", lo cual sugiere que la especie se enfrenta a un riesgo alto de extinción en su estado silvestre, debido principalmente a su distribución restringida y bajo número de individuos que componen sus poblaciones (Hechenleitner et al. 2005). Es una especie monotípica y endémica de los bosques esclerófilos y de Nothofagus de la zona mediterránea de Chile, circunscrita a la precordillera y cordillera de los Andes 
(Rodríguez et al. 1983) entre los $35^{\circ} 16^{\prime}$ y $36^{\circ} 41^{\prime}$ S (Hechenleitner et al. 2005). El tamaño y forma de las semillas es variable, y su dispersión es a través del agua o gravedad, característica que repercute en su limitada dispersión a grandes distancias (Hechenleitner et al. 2005). De acuerdo con los mismos autores ninguna de las poblaciones identificadas de Legrandia se encuentra presente en áreas protegidas privadas o del estado.

El objetivo de este estudio es evaluar el efecto de la fragmentación sobre poblaciones de Legrandia mediante el uso de índices de paisaje y variables de estructura de la vegetación. En particular, se describe el patrón de ocupación de las poblaciones de Legrandia y se evalúan las relaciones entre tamaño, forma, nivel de aislamiento y distancia al borde en parches con presencia de Legrandia y variables de estructura de la vegetación. En este trabajo nosotros hipotetizamos que en fragmentos de menor tamaño, formas regulares y altos niveles de aislamiento la presencia de la especie decrecerá, manifestándose una menor abundancia, tanto de árboles, brinzales y plántulas. Además, creemos que la presencia de la especie también se ve afectada negativamente, mientras menor sea la distancia entre la población y el borde del fragmento. Este es el primer estudio en poblaciones amenazadas de Chile que relaciona atributos espaciales medidos mediante imágenes satelitales e información de estructura de la vegetación obtenida en terreno. Este trabajo aporta nuevos antecedentes para el estudio de poblaciones con problemas de conservación, mediante el uso de nuevas aproximaciones, lo cual permite proponer medidas de conservación para la especie.

\section{MATERIALES Y MÉTODOS}

\section{Área de estudio}

El área de estudio fue establecida a partir de los límites longitudinales y altitudinales de distribución que señala la literatura para esta especie, es decir, entre los $35^{\circ} 16^{\prime}$ y $36^{\circ} 41^{\prime}$ S, y entre 400 y $1.000 \mathrm{~m}$ de altitud (Landrum 1986, Hechenleitner et al. 2005, Martínez et al. en prensa) (Fig. 1). El área total de estudio corresponde a 688.916 ha, y ocupa parte de la zona andina de la VII y VIII región administrativa de Chile (Fig. 1). El clima predominante corresponde al tipo mediterráneo, caracterizado por precipitaciones concentradas en el invierno entre aproximadamente $1.500 \mathrm{y}$ $2.100 \mathrm{~mm}$ anuales y temperatura media anual entre 11,8 y $13{ }^{\circ} \mathrm{C}$ (Lara et al. 2001, Pezoa 2003). La zona andina de las regiones administrativas de este estudio se caracteriza por la presencia de bosques nativos dominados por especies tales como Nothofagus obliqua, $N$. glauca, $N$. dombeyi además de plantaciones industriales de especies forestales y bosques mixtos de especies nativas y exóticas (CONAF et al. 1999). Legrandia se presenta en estos ecosistemas formando rodales relativamente puros o bajo un dosel de árboles de mayor tamaño de Nothofagus obliqua, N. glauca o $N$. dombeyi (Martínez et al. en prensa). Esta especie es un pequeño árbol de la familia Myrtaceae, que no supera los $8 \mathrm{~m}$ de altura (Serra et al. 1986). Su fruto corresponde a una baya, de gran tamaño en relación a otras Mirtáceas, generalmente redondeada de $1,5-2 \mathrm{~cm}$ de diámetro (Rodríguez et al. 1983). La forma de regeneración es principalmente a través de raíz y tocón, y no se ha evidenciado regeneración por semillas (Martínez et al. en prensa).

\section{Información de terreno}

Se recolectó información georreferenciada sobre la ubicación de 28 poblaciones de Legrandia, las cuales se distribuían en 12 fragmentos de bosque nativo (Fig. 1 y Tabla 1). De estos, nueve fragmentos fueron seleccionados para su muestreo en terreno. Los criterios de selección consideraron los rangos de latitud, altitud, tamaño de fragmentos y accesibilidad. Un total de 10 parcelas de 400 $\mathrm{m}^{2}(20 \times 20 \mathrm{~m})$ se establecieron en los fragmentos con presencia de Legrandia. Árboles (Dap $\geq 5 \mathrm{~cm}$ ) y brinzales (Dap $<5 \mathrm{~cm}$ y altura $\geq 1,3 \mathrm{~m}$ ) de todas las especies fueron medidos en cada parcela, y para contabilizar la presencia de plántulas (altura $<1,3 \mathrm{~m}$ ) se establecieron 12 subparcelas de $4 \mathrm{~m}^{2}(2 \times 2 \mathrm{~m})$ en cada una de ellas. También fueron medidas variables de sitio como altitud, exposición y pendiente. En aquellos fragmentos con más de una población presente y de tamaño mayor a 500 ha se establecieron dos parcelas de muestreo. 


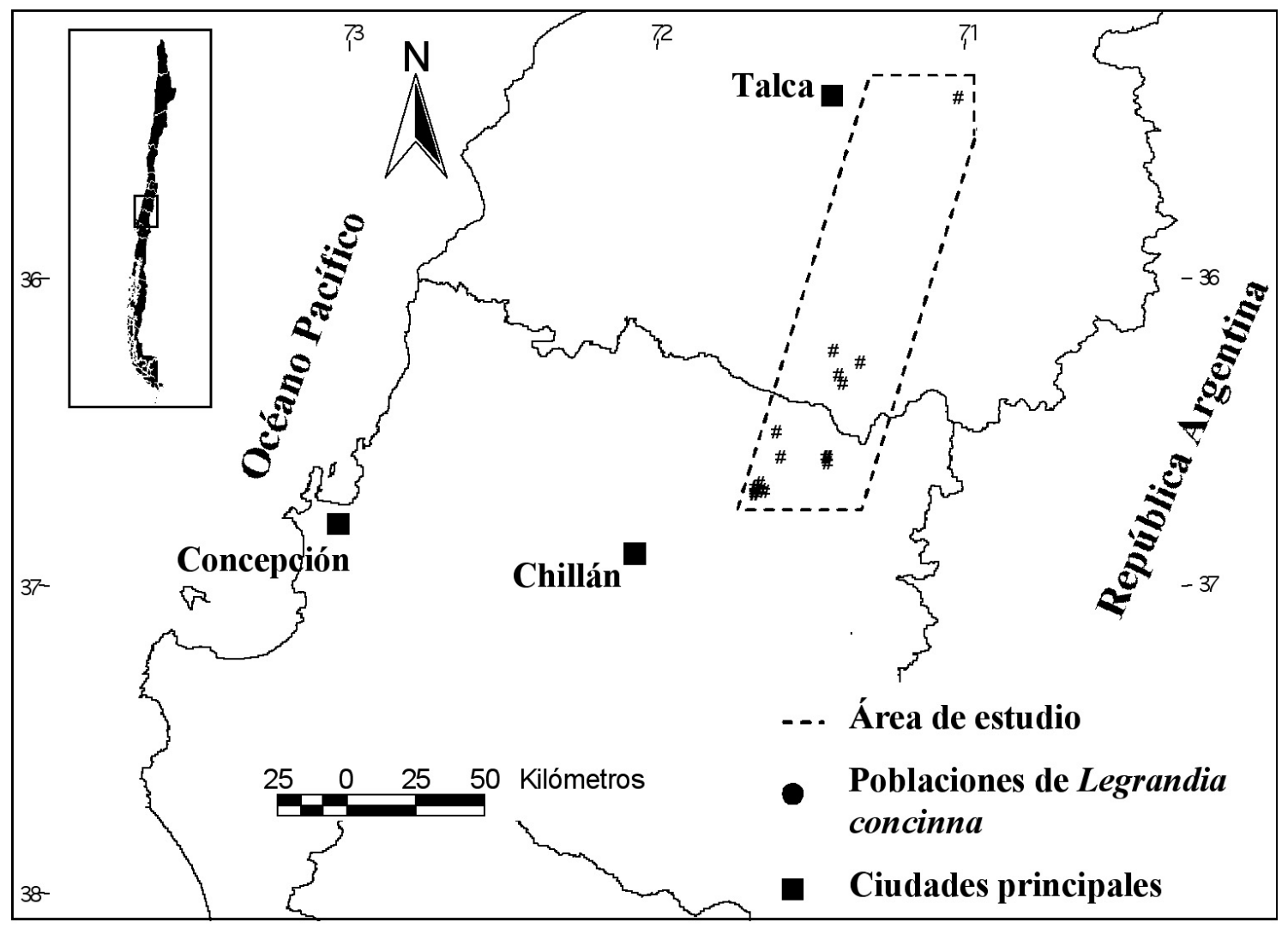

Fig. 1: Distribución de poblaciones de Legrandia en el área de estudio.

Distribution of Legrandia populations included in study area.

Procesamiento y clasificación de imagen satelital

A fin de clasificar la cobertura boscosa en el área de estudio y calcular los índices de paisaje de los fragmentos con presencia y sin presencia de Legrandia se utilizó una imagen satelital Landsat 7 ETM+ (Enhanced Thematic Mapper), del 29 de enero de 2001 (Path 233, Row 85). También fueron utilizadas coberturas vectoriales de cursos de agua, caminos y usos del suelo a escala 1:50.000 (CONAF et al. 1999). La imagen fue corregida geométrica y atmosféricamente siguiendo los procedimientos tradicionales (Lillesand \& Kiefer 1994). Se aplicó una clasificación de tipo supervisada, en la cual se asignan áreas de entrenamiento representativas de los tipos de coberturas del suelo que compondrán la clasificación (Mather 1999). Los tipos de coberturas del suelo definidas en la clasificación fueron: bosque nativo (principalmente renovales de Nothofagus obliqua, N. glauca, N. alessandri, y bosquetes del tipo esclerófilo sobre $2 \mathrm{~m}$ de altura y $>50$ $\%$ de cobertura), matorrales (compuestos principalmente por especies esclerófilas de menor altura tales como Criptocarya alba, Quillaja saponaria y Lithraea caustica), plantaciones exóticas (principalmente de la especie Pinus radiata), terrenos de uso agrícola, herbáceas y praderas, y otros usos (terrenos sin vegetación, áreas urbanas y cuerpos de agua). El criterio o regla de clasificación utilizada fue el de Máxima Verosimilitud, el cual es el método más utilizado en estudios de sensores remotos (Mather 1999). Para evaluar la separabilidad espectral de las áreas de entrenamiento se utilizó el índice de divergencia transformado, el cual es recomendado por ERDAS (1999). La evaluación de la exactitud de la clasificación se realizó mediante una matriz de confusión o errores (Lillesand \& Kiefer 1994). Para el tratamiento de la imagen satelital se utilizó el programa ERDAS Imagine 8.4®. 


\section{TABLA 1}

Ubicación de poblaciones de Legrandia incluidas en el estudio

Location of Legrandia populations included in the study area

\begin{tabular}{|c|c|c|c|c|c|}
\hline Población ${ }^{1}$ & Código & Provincia & Latitud S & Longitud $\mathrm{O}$ & Fuente \\
\hline Bullileo & BULL & Linares & $36^{\circ} 20^{\prime} 31,92^{\prime \prime}$ & $71^{\circ} 24^{\prime} 13,75^{\prime \prime}$ & Proyecto Darwin ${ }^{2,3}$ \\
\hline Coihueco1 & $\mathrm{CO} 1$ & Ñuble & $36^{\circ} 34^{\prime} 47,93^{\prime \prime}$ & $71^{\circ} 36^{\prime} 07,42^{\prime \prime}$ & Forestal Bío Bío S.A. ${ }^{3}$ \\
\hline Coihueco2 & $\mathrm{CO} 2$ & Nuble & $36^{\circ} 40^{\prime} 52,28^{\prime \prime}$ & $71^{\circ} 41^{\prime} 07,80^{\prime \prime}$ & Forestal Bío Bío S.A. \\
\hline Coihueco3 & $\mathrm{CO} 3$ & Ñuble & $36^{\circ} 40^{\prime} 50,41^{\prime \prime}$ & $71^{\circ} 41^{\prime} 09,31^{\prime \prime}$ & Forestal Bío Bío S.A. ${ }^{3}$ \\
\hline Coihueco4 & $\mathrm{CO} 4$ & Ñuble & $36^{\circ} 40^{\prime} 50,30^{\prime \prime}$ & $71^{\circ} 41^{\prime} 07,87^{\prime \prime}$ & Forestal Bío Bío S.A. \\
\hline Coihueco5 & $\mathrm{CO} 5$ & Ñuble & $36^{\circ} 40^{\prime} 51,46^{\prime \prime}$ & $71^{\circ} 41^{\prime} 07,87^{\prime \prime}$ & Forestal Bío Bío S.A. \\
\hline Coihueco6 & $\mathrm{CO} 6$ & Ñuble & $36^{\circ} 40^{\prime} 52,61^{\prime \prime}$ & $71^{\circ} 41^{\prime} 07,76^{\prime \prime}$ & Forestal Bío Bío S.A. \\
\hline Coihueco7 & $\mathrm{CO} 7$ & Ñuble & $36^{\circ} 40^{\prime} 53,22^{\prime \prime}$ & $71^{\circ} 41^{\prime} 07,26^{\prime \prime}$ & Forestal Bío Bío S.A. \\
\hline Coihueco8 & $\mathrm{CO} 8$ & Ñuble & $36^{\circ} 40^{\prime} 53,47^{\prime \prime}$ & $71^{\circ} 41^{\prime} 06,32^{\prime \prime}$ & Forestal Bío Bío S.A. \\
\hline Coihueco9 & $\mathrm{CO} 9$ & Ñuble & $36^{\circ} 40^{\prime} 53,47^{\prime \prime}$ & $71^{\circ} 40^{\prime} 54,41^{\prime \prime}$ & Forestal Bío Bío S.A. \\
\hline Coihueco10 & $\mathrm{CO} 10$ & Ñuble & $36^{\circ} 41^{\prime} 14,21^{\prime \prime}$ & $71^{\circ} 39^{\prime} 57,10^{\prime \prime}$ & Forestal Bío Bío S.A. \\
\hline Coihueco11 & CO11 & Ñuble & $36^{\circ} 41^{\prime} 40,96^{\prime \prime}$ & $71^{\circ} 41^{\prime} 11,33^{\prime \prime}$ & Forestal Bío Bío S.A. ${ }^{3}$ \\
\hline Coihueco12 & $\mathrm{CO} 12$ & Ñuble & $36^{\circ} 41^{\prime} 40,42^{\prime \prime}$ & $71^{\circ} 41^{\prime} 08,95^{\prime \prime}$ & Forestal Bío Bío S.A. \\
\hline Coihueco13 & $\mathrm{CO} 13$ & Ñuble & $36^{\circ} 41^{\prime} 40,20^{\prime \prime}$ & $71^{\circ} 41^{\prime} 08,48^{\prime \prime}$ & Forestal Bío Bío S.A. \\
\hline Coihueco14 & $\mathrm{CO} 14$ & Ñuble & $36^{\circ} 42^{\prime} 04,32^{\prime \prime}$ & $71^{\circ} 41^{\prime} 02,36^{\prime \prime}$ & Forestal Bío Bío S.A. \\
\hline Coihueco15 & $\mathrm{CO} 15$ & Ñuble & $36^{\circ} 42^{\prime} 00,79^{\prime \prime}$ & $71^{\circ} 41^{\prime} 21,95^{\prime \prime}$ & Forestal Bío Bío S.A. \\
\hline Coihueco16 & $\mathrm{CO} 16$ & Ñuble & $36^{\circ} 42^{\prime} 00,86^{\prime \prime}$ & $71^{\circ} 41^{\prime} 17,16^{\prime \prime}$ & Forestal Bío Bío S.A. \\
\hline Coihueco17 & $\mathrm{CO} 17$ & Ñuble & $36^{\circ} 42^{\prime} 03,60^{\prime \prime}$ & $71^{\circ} 41^{\prime} 17,20^{\prime \prime}$ & Forestal Bío Bío S.A. ${ }^{3}$ \\
\hline Coihueco18 & $\mathrm{CO} 18$ & Ñuble & $36^{\circ} 39^{\prime} 55,30^{\prime \prime}$ & $71^{\circ} 40^{\prime} 09,26^{\prime \prime}$ & Herbario de Concepción ${ }^{3}$ \\
\hline La Balsa & LABA & Linares & $36^{\circ} 16^{\prime} 25,21^{\prime \prime}$ & $71^{\circ} 20^{\prime} 44,022$ & Proyecto Darwin ${ }^{3}$ \\
\hline Minas del Prado & MPRA & Ñuble & $36^{\circ} 41^{\prime} 28,36^{\prime}$ & $71^{\circ} 39^{\prime} 18,40^{\prime \prime}$ & Proyecto Darwin \\
\hline Parral1 & PAR1 & Linares & $36^{\circ} 14^{\prime} 05,71^{\prime \prime}$ & $71^{\circ} 25^{\prime} 55,63^{\prime \prime}$ & Herbario de Concepción \\
\hline Parral2 & PAR2 & Linares & $36^{\circ} 19^{\prime} 01,78^{\prime \prime}$ & $71^{\circ} 25^{\prime} 00,12^{\prime \prime}$ & Herbario de Concepción \\
\hline Radal & RADA & Curicó & $35^{\circ} 24^{\prime} 40,03^{\prime \prime}$ & $71^{\circ} 01^{\prime} 28,52^{\prime \prime}$ & Proyecto Darwin ${ }^{3}$ \\
\hline San Fabián1 & SFB 1 & Ñuble & $36^{\circ} 29^{\prime} 54,17^{\prime \prime}$ & $71^{\circ} 36^{\prime} 56,95^{\prime \prime}$ & Herbario de Concepción ${ }^{3}$ \\
\hline San Fabián2 & SFB2 & Ñuble & $36^{\circ} 35^{\prime} 00,02^{\prime \prime}$ & $71^{\circ} 27^{\prime} 00,04^{\prime \prime}$ & Herbario de Concepción \\
\hline San Fabián3 & SFB3 & Ñuble & $36^{\circ} 36^{\prime} 00,00^{\prime \prime}$ & $71^{\circ} 27^{\prime} 00,00^{\prime \prime}$ & Herbario de Concepción \\
\hline San Fabián4 & SFB4 & Ñuble & $36^{\circ} 34^{\prime} 41,16^{\prime \prime}$ & $71^{\circ} 27^{\prime} 18,97^{\prime \prime}$ & Proyecto Darwin \\
\hline
\end{tabular}

${ }^{1}$ Nombre dado por la localidad más cercana;

2 Proyecto "Programa de conservación integrado para las especies forestales endémicas amenazadas de Chile"; 162/11/012. Financiado por Darwin Initiative del Departamento de Medio Ambiente del Reino Unido y ejecutado por el Instituto de Silvicultura de la Universidad Austral de Chile;

${ }^{3}$ Visitadas y medidas en terreno por los autores.

\section{Cálculo de índices de paisaje}

Mediante la revisión de estudios recientes de fragmentación de bosques (Gigord et al. 1999, Franklin 2001, Armenteras et al. 2003, Fitzsimmons 2003, Echeverría et al. 2006) se seleccionaron los siguientes índices de paisaje, los cuales fueron calculados para fragmentos de bosque nativo con presencia y sin presencia de poblaciones de Legrandia: área del fragmento (ha), índice de proximidad media (cuociente entre el área del fragmento y la proximidad de todos los fragmentos cuyos bordes están dentro de un rango específico de distancia de búsqueda del fragmento) e índice de forma (cuociente entre el perímetro del fragmento y el mínimo perímetro que puede tener el fragmento simulando una forma regular). Índice de proximidad media (IPM) fue calculado como medida del aislamiento de los fragmentos bajo 
una distancia de búsqueda de 100,500 y 1.000 $\mathrm{m}$. Aunque se trata de un índice no normalizado (es decir, no varía entre 0 y 1 ) devuelve valores absolutos. Los valores bajos indican fragmentos que están relativamente aislados de otros fragmentos dentro de la distancia especificada, mientras que altos valores indican parches que están relativamente conectados con otros parches (Gustafson \& Parker 1992, Venema et al. 2005). IPM fue calculado mediante la siguiente fórmula (Mcgarigal \& Marks 1995):

$$
I P M=\sum_{i=1}^{n} \frac{a_{i}}{h_{i}^{2}}
$$

Donde,

$a_{i}=$ Área del fragmento i

$h_{i}{ }^{2}=$ Distancia al cuadrado al vecino i.

El índice de forma (IFO) fue estimado mediante la siguiente fórmula (McGarigal \& Marks 1995):

$$
I F O=\frac{p_{i}}{\min \rightarrow p_{i}}
$$

Donde,

$p_{i}=$ Perímetro del fragmento $\mathrm{i}$

mín $\rightarrow p_{i=}$ Mínimo perímetro del fragmento i

IFO puede tomar valores mayores o iguales a 1 . El valor 1 es alcanzado en fragmentos máximamente compactos o regulares (como un cuadrado o círculo) y aumenta su valor sin límite en fragmentos de forma más irregular.

Para obtener una medida de cada población dentro del fragmento fue calculada la distancia al borde del fragmento (DBF) de cada población. DBF fue calculado como la distancia en metros entre la coordenada geográfica de cada población y el borde del pixel más cercano del fragmento. Este índice solo fue aplicado a fragmentos con presencia de poblaciones de Legrandia, y no a otros fragmentos sin presencia de la especie. Para el análisis espacial fueron utilizados los programas ARCVIEW 3.2® y FRAGSTATS 3.3 (Mcgarigal \& Marks 1995).

\section{Análisis de datos}

(A) Patrón de ocupación en relación a índices de paisaje: área, aislamiento y forma. Se seleccionó en forma estratificada 162 fragmentos con presencia y sin presencia de Legrandia en el área de estudio (150 sin presencia y 12 con presencia comprobada de Legrandia). Se analizó la presencia o uso relativo de las poblaciones de acuerdo con el tamaño, aislamiento (IPM) y forma (IFO) de los fragmentos, de manera similar a funciones de incidencia utilizadas en otros estudios (Verboom \& van Apeldoorn 1990, Virgós \& García 2002). Cada índice de paisaje fue clasificado en seis categorías respecto a tamaño, aislamiento (IPM) y forma (IFO).

En forma aleatoria, a partir de la muestra de 162 fragmentos, se seleccionaron 15 fragmentos con presencia y sin presencia de Legrandia por cada categoría de índice de paisaje (90 fragmentos en total). El uso relativo de las distintas categorías fue expresado como porcentaje entre el número de fragmentos con presencia de Legrandia dividido por el número total de fragmentos seleccionados en cada categoría. Se comparó la distribución de frecuencias de los fragmentos sin presencia (150 fragmentos seleccionados aleatoriamente) y con presencia (12 fragmentos) de Legrandia utilizando pruebas no paramétricas de Kolmogorov-Smirnov (Dytham 2003).

(B) Correlaciones entre variables de estructura de la vegetación e índices de paisaje. Con el objetivo de eliminar la autocolinearidad entre los índices de paisaje se aplicaron análisis de correlación simple a fragmentos sin presencia de Legrandia seleccionados aleatoriamente. Posteriormente, fueron seleccionados los índices más relevantes para ser relacionados con las variables vegetacionales.

Para determinar las variables vegetacionales que contribuyen mayormente a explicar la variación de los datos fue realizado un análisis de componentes principales (PCA) y un análisis de correlación simple.

Para relacionar variables que miden el efecto de la fragmentación con variables de estructura de la vegetación de poblaciones de Legrandia, se aplicaron análisis de correlación simple entre los índices de paisaje y las variables de estructura de la vegetación medidas en terreno. Debido a la naturaleza de los datos, en todos los análisis de correlación fueron utilizadas pruebas no paramétricas de Spearman (Dytham 2003). 


\section{RESULTADOS}

Precisión de clasificación de imagen satelital y cobertura actual del suelo

El índice de divergencia transformado varió entre 1890 y 2000 en los tipos de coberturas del suelo, lo cual de acuerdo con ERDAS (1999) indica una buena separabilidad espectral de las áreas de entrenamiento. La exactitud global de la clasificación alcanzó un $91 \%$, presentando para cada una de las categorías valores superiores a $80 \%$.

La distribución actual de la cobertura del suelo del área de estudio corresponde a: bosque nativo (30\%), matorrales (17\%), plantaciones exóticas $(8 \%)$, terrenos de uso agrícola $(7 \%)$, herbáceas y praderas $(15 \%)$. La categoría "otros usos" que agrupa a terrenos sin vegetación, áreas urbanas y cuerpos de agua representa un $23 \%$ del área de estudio.

Índices de paisaje en fragmentos de bosque nativo sin presencia de Legrandia

La distribución de frecuencias del área de fragmentos de bosque nativo sin presencia de Legrandia muestra que una alta proporción (70 \%) de estos son de tamaño inferior a 1 ha (Fig. 2). El índice de proximidad media (IPM) no mostró diferencias sustanciales al ser calculado a diferentes distancias de búsqueda. Debido a esto, se utilizó una medida intermedia de $500 \mathrm{~m}$. IPM muestra una importante concentración de los fragmentos de bosque nativo sin presencia de Legrandia en valores inferiores, lo cual indica que una proporción importante de estos fragmentos presenta altos niveles de aislamiento (Fig. 2).
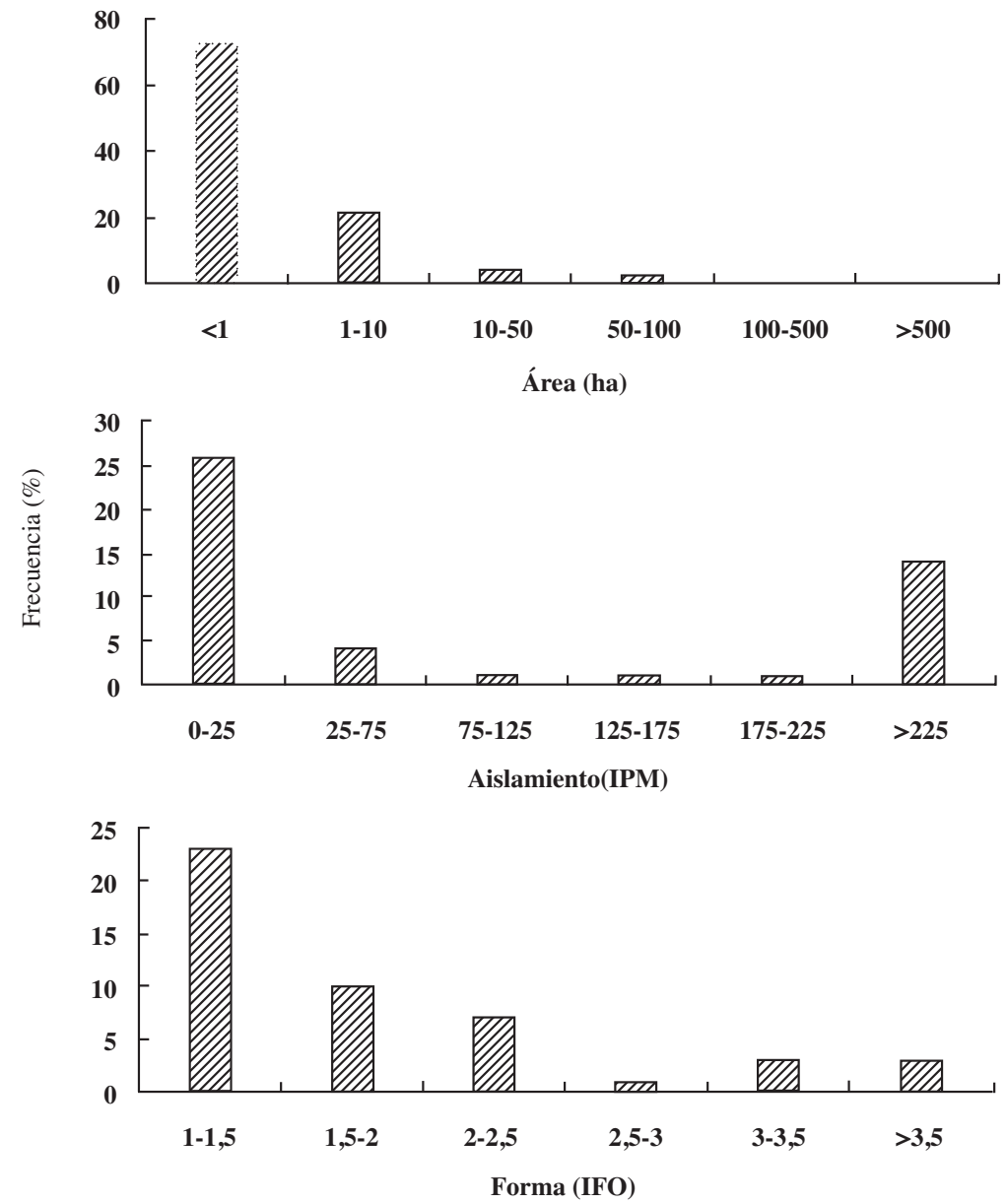

Fig. 2: Frecuencia de fragmentos de bosque nativo sin presencia de Legrandia en relación a área, aislamiento (IPM) y forma (IFO) $(\mathrm{n}=150)$.

Frequency of native forest fragments with absence of Legrandia related to area, isolation (IPM) and shape (IFO) $(\mathrm{n}=150)$. 
Al analizar la correlación entre los índices de paisaje, solo se presenta una correlación positiva y significativa entre área e índice de forma (IFO) (prueba de Spearman, $\mathrm{r}_{\mathrm{s}}=0,74 ; \mathrm{P}$ $<0,001$ ) (Tabla 3). Debido a esto último fue seleccionada área entre estos dos índices para evaluar el patrón de ocupación y las relaciones con variables de estructura de la vegetación. Ello puesto que área o tamaño del fragmento es un índice de fácil comprensión y más utilizado en estudios de fragmentación (Gigord et al. 1999, Armenteras et al. 2003, Fitzsimmons 2003, Echeverría et al. 2006).

Patrón de ocupación de Legrandia en relación a tamaño y aislamiento de fragmentos

Los resultados mostraron que un $60 \%$ de los fragmentos con presencia de la especie corresponden a fragmentos menores a 10 ha (Fig. 3). Respecto al IPM un $75 \%$ de los fragmentos con presencia de Legrandia se presentan en las categorías de mayor aislamiento (Fig. 3). Al comparar la distribución de frecuencias tanto para área como para IPM entre fragmentos con presencia y sin presencia de Legrandia no se observan diferencias significativas (prueba de Kolmogorov-Smirnov, $\mathrm{K}-\mathrm{S}=0,57 ; \mathrm{P}=0,89$ ) (Fig. 4). Esto significa que con los datos de estudio no existen evidencias para afirmar que los fragmentos de bosque nativo sin presencia de Legrandia presentan un patrón de distribución distinto a los fragmentos con presencia de Legrandia, respecto a tamaño y aislamiento.

Variables de estructura de la vegetación y sitio

La mayor parte de las variables de estructura de la vegetación estuvieron correlacionadas entre ellas (Tabla 4). La densidad de árboles de Legrandia presentó una correlación positiva y

TABLA 2

Variables de estructura de la vegetación y sitio en poblaciones de Legrandia medidas en terreno $(\mathrm{n}=10)$. Ver Tabla 1 para códigos de poblaciones

Vegetation structure and site variables of Legrandia populations measured in the field $(\mathrm{n}=10)$. See Table 1 for the codes of the study populations

\begin{tabular}{|c|c|c|c|c|c|c|c|c|c|c|}
\hline Variable & RADA & LABA & BULL & SFB 1 & $\mathrm{CO} 1$ & $\mathrm{CO} 18$ & $\mathrm{CO} 3$ & $\mathrm{CO} 9$ & CO11 & $\mathrm{CO} 17$ \\
\hline Densidad total árboles* $\left(\mathrm{N} \mathrm{ha}^{-1}\right)$ & 625 & 1.775 & 1.525 & 800 & 1.000 & 650 & 350 & 925 & 2.425 & 1.600 \\
\hline Densidad árboles Legrandia $\left(\mathrm{N} \mathrm{ha}^{-1}\right)$ & 75 & 1.200 & 625 & 300 & 225 & 150 & 125 & 150 & 775 & 200 \\
\hline Densidad total brinzales $\uparrow\left(\mathrm{N} \mathrm{ha}^{-1}\right)$ & 950 & 2.025 & 2.100 & 1.100 & 1.300 & 425 & 200 & 1.575 & 2.150 & 3.850 \\
\hline Densidad brinzales Legrandia $\left(\mathrm{N} \mathrm{ha}^{-1}\right)$ & 75 & 0 & 1.050 & 200 & 550 & 175 & 50 & 525 & 1.600 & 2.000 \\
\hline Densidad total plántulas $\$\left(\mathrm{~N} \mathrm{ha}^{-1}\right)$ & 3.125 & 3.333 & 3.958 & 7.292 & 5.625 & 2.292 & 2.500 & 1.458 & 10.208 & 2.083 \\
\hline Densidad plántulas Legrandia $\left(\mathrm{N} \mathrm{ha}^{-1}\right)$ & 208 & 2.083 & 1.875 & 5.000 & 3.125 & 1.458 & 1.042 & 833 & 5.833 & 1.458 \\
\hline Área basal total $\left(\mathrm{m}^{2} \mathrm{ha}^{-1}\right)$ & 14,4 & 26,0 & 18,2 & 42,7 & 18,0 & 6,2 & 5,3 & 7,1 & 42,2 & 14,4 \\
\hline Área basal Legrandia $\left(\mathrm{m}^{2} \mathrm{ha}^{-1}\right)$ & 0,5 & 11,1 & 5,1 & 3,3 & 1,6 & 0,7 & 0,6 & 0,9 & 6,2 & 0,4 \\
\hline Pendiente $(\%)$ & 25 & 25 & 3 & 40 & 25 & 30 & 35 & 10 & 30 & 1 \\
\hline Altitud (m) & 720 & 955 & 700 & 1.100 & 643 & 610 & 550 & 750 & 870 & 911 \\
\hline Exposición & S-E & W & W & E & N-W & W & W & W & W & V \\
\hline
\end{tabular}

* Dap $\geq 5 \mathrm{~cm} ; \dagger$ Dap $<5 \mathrm{~cm}$ y altura $\geq 1,3 \mathrm{~m} ; \ddagger$ altura $<1,3 \mathrm{~m}$

TABLA 3

Correlación para índices de paisaje en fragmentos sin presencia de Legrandia (prueba de Spearman, $\mathrm{n}=150$ )

Correlation of landscape indices in fragments with absence of Legrandia (Spearman's test, n $=150$ )

\begin{tabular}{lcc}
\hline Índice de paisaje & Índice de proximidad media (IPM) & Índice de forma (IFO) \\
\hline Área & $\mathrm{r}_{\mathrm{s}}=0,138 \mathrm{P}=0,354$ & $\mathrm{r}_{\mathrm{s}}=0,740 \mathrm{P}<0,001$ \\
Índice de proximidad media (IPM) & - & $\mathrm{r}_{\mathrm{s}}=0,032 \mathrm{P}=0,833$ \\
\hline
\end{tabular}




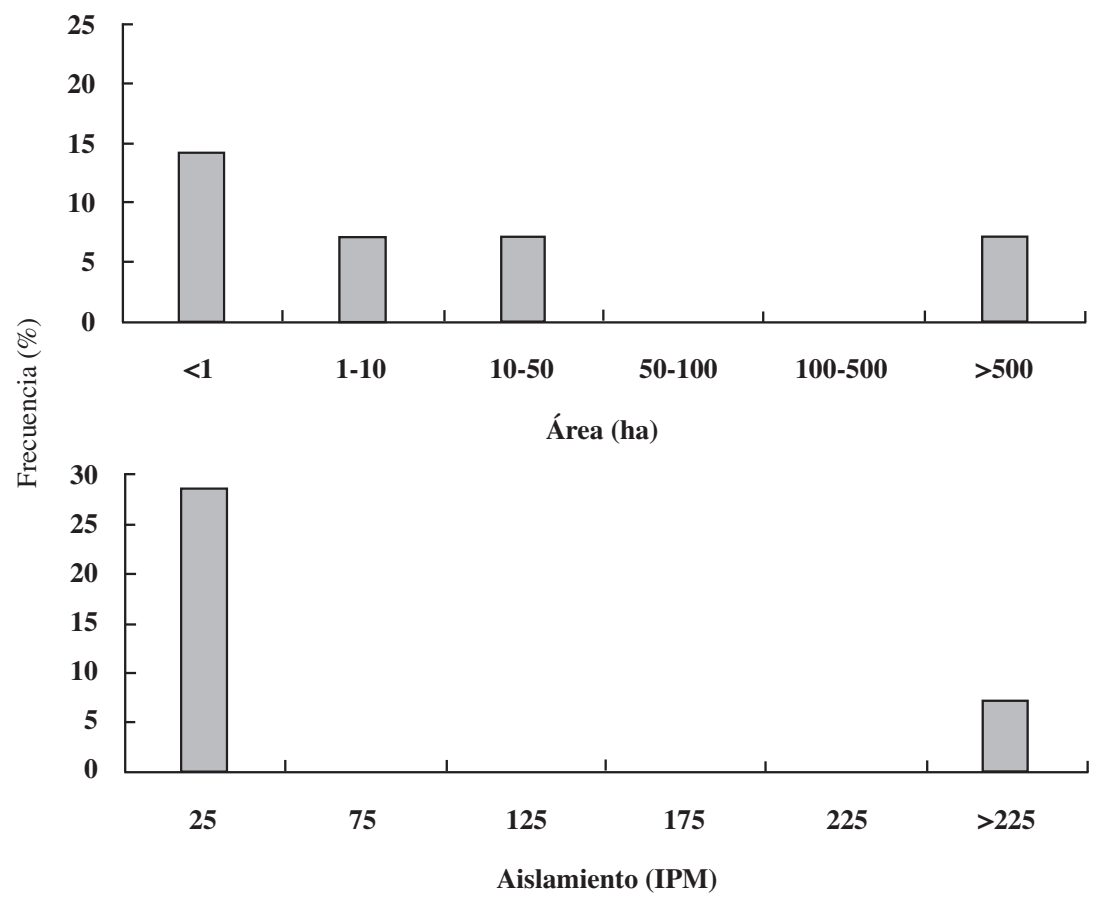

Fig. 3: Patrón de ocupación de poblaciones de Legrandia en relación a área y aislamiento (IPM) $(\mathrm{n}=90)$. Occupancy pattern of Legrandia populations related to area and isolation (IPM) $(\mathrm{n}=90)$.

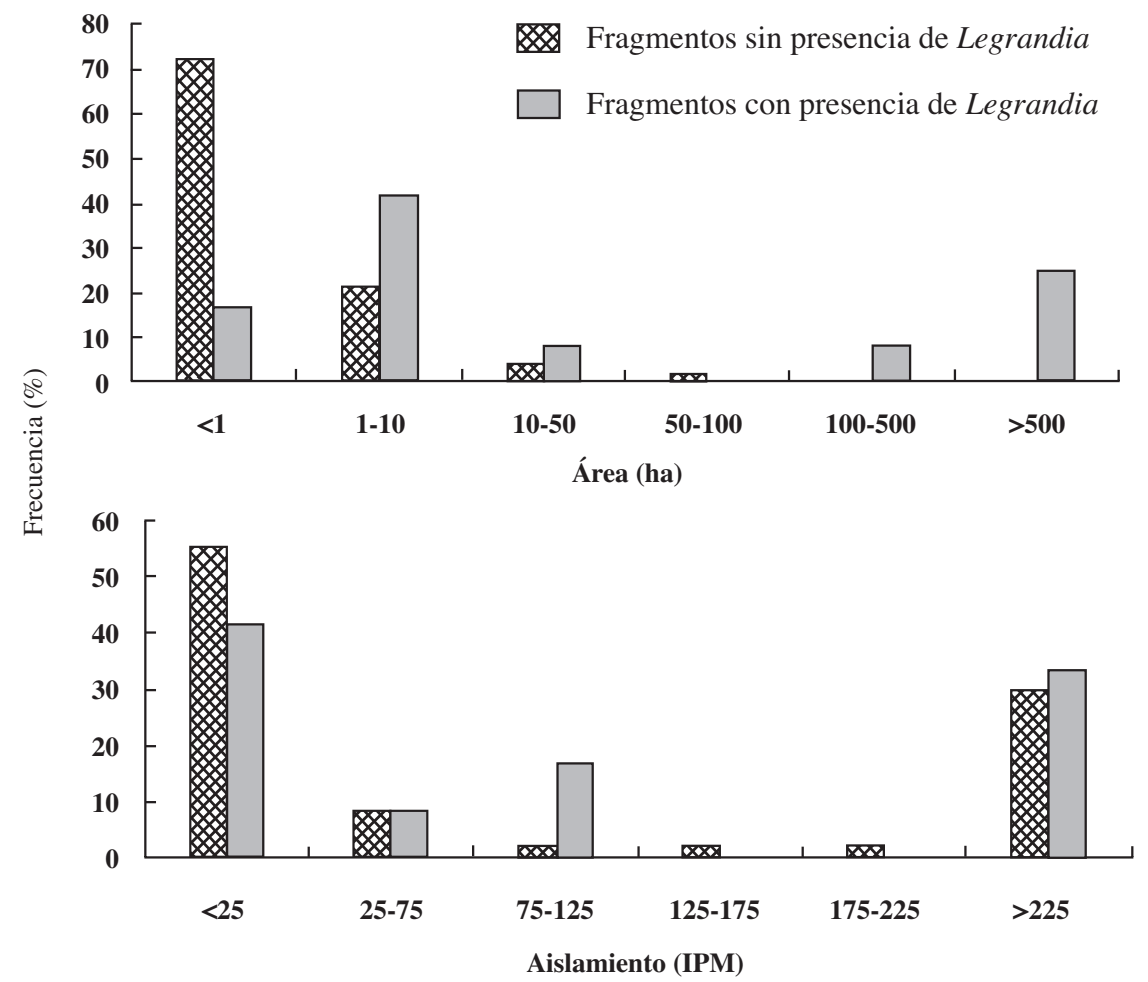

Fig. 4: Frecuencia de fragmentos de bosque nativo con presencia y sin presencia de Legrandia en relación a área y aislamiento $($ IPM $)(\mathrm{n}=162)$.

Frequency of native forest fragments with and without Legrandia presence related to area and isolation (IPM) $(\mathrm{n}=162)$. 


\section{TABLA 4}

Correlación entre variables de estructura de la vegetación y sitio en poblaciones de Legrandia (prueba de Spearman, $\mathrm{n}=10$ ). Correlaciones estadísticamente significativas indicadas en "negritas"

Correlation between vegetation structure and site variables in Legrandia populations (Spearman's test, $\mathrm{n}=10$ ). Statistically significant correlations are indicated in bold typing

\begin{tabular}{|c|c|c|c|c|c|c|c|c|c|}
\hline Variable & DAL & DTB & DBL & DTP & DPL & $\mathrm{ABT}$ & $\mathrm{ABL}$ & PEN & ALT \\
\hline Densidad total árboles (DA) & $\begin{array}{l}r_{s}=0,845 \\
P=0,002\end{array}$ & $\begin{array}{l}r_{s}=0,915 \\
P=0,001\end{array}$ & $\begin{array}{l}r_{s}=0,539 \\
P=0,108\end{array}$ & $\begin{array}{l}P=0,328 \\
P=0,328\end{array}$ & $\begin{array}{l}r_{s}=0,620 \\
P=0,056\end{array}$ & $\begin{array}{l}\mathrm{r}_{\mathrm{s}}=0,588 \\
\mathrm{P}=0,074\end{array}$ & $\begin{array}{l}r_{s}=0,600 \\
P=0,067\end{array}$ & $\begin{array}{l}r_{s}=-0,389 \\
P=0,267\end{array}$ & $\begin{array}{l}r_{s}=0,552 \\
P=0,098\end{array}$ \\
\hline Densidad árboles Legrandia (DAL) & - & $\begin{array}{l}r_{s}=0,650 \\
P=0,042\end{array}$ & $\begin{array}{l}r_{s}=0,261 \\
P=0,466\end{array}$ & $\begin{array}{l}r_{s}=0,626 \\
P=0,053\end{array}$ & $\begin{array}{l}r_{s}=0,838 \\
P=0,002\end{array}$ & $\begin{array}{l}r_{s}=0,790 \\
P=0,007\end{array}$ & $\begin{array}{l}r_{s}=0,875 \\
P=0,001\end{array}$ & $\begin{array}{l}r_{s}=-0,081 \\
P=0,825\end{array}$ & $\begin{array}{l}r_{s}=0,553 \\
P=0,097\end{array}$ \\
\hline Densidad total brinzales (DTB) & & - & $\begin{array}{l}r_{s}=0,721 \\
P=0,019\end{array}$ & $\begin{array}{l}r_{s}=0,152 \\
P=0,676\end{array}$ & $\begin{array}{l}r_{s}=0,395 \\
P=0,258\end{array}$ & $\begin{array}{l}r_{s}=0,467 \\
P=0,174\end{array}$ & $\begin{array}{l}r_{s}=0,333 \\
P=0,347\end{array}$ & $\begin{array}{l}r_{s}=-0,593 \\
P=0,071\end{array}$ & $\begin{array}{l}r_{s}=0,564 \\
P=0,090\end{array}$ \\
\hline Densidad brinzales Legrandia (DBL) & & & - & $\begin{array}{l}r_{s}=0,152 \\
P=0,676\end{array}$ & $\begin{array}{l}r_{s}=0,395 \\
P=0,258\end{array}$ & $\begin{array}{l}r_{s}=0,212 \\
P=0,556\end{array}$ & $\begin{array}{l}r_{s}=-0,030 \\
P=0,934\end{array}$ & $\begin{array}{l}r_{s}=-0,420 \\
P=0,227\end{array}$ & $\begin{array}{l}r_{s}=0,164 \\
P=0,651\end{array}$ \\
\hline Densidad total plántulas (DTP) & & & & - & $\begin{array}{l}r_{s}=0,827 \\
P=0,003\end{array}$ & $\begin{array}{l}r_{s}=0,830 \\
P=0,003\end{array}$ & $\begin{array}{l}r_{s}=0,673 \\
P=0,033\end{array}$ & $\begin{array}{l}r_{s}=0,377 \\
P=0,283\end{array}$ & $\begin{array}{l}r_{s}=0,248 \\
P=0,489\end{array}$ \\
\hline Densidad plántulas Legrandia (DPL) & & & & & - & $\begin{array}{l}r_{s}=0,784 \\
P=0,007\end{array}$ & $\begin{array}{l}r_{s}=0,729 \\
P=0,017\end{array}$ & $\begin{array}{l}r_{s}=0,316 \\
P=0,374\end{array}$ & $\begin{array}{l}r_{s}=0,407 \\
P=0,243\end{array}$ \\
\hline Área basal total (ABT) & & & & & & - & $\begin{array}{l}r_{s}=0,733 \\
P=0,016\end{array}$ & $\begin{array}{l}r_{s}=0,105 \\
P=0,773\end{array}$ & $\begin{array}{l}r_{s}=0,709 \\
P=0,022\end{array}$ \\
\hline Área basal Legrandia (ABL) & & & & & & & - & $\begin{array}{l}r_{s}=0,062 \\
P=0,865\end{array}$ & $\begin{array}{l}r_{s}=0,358 \\
P=0,310\end{array}$ \\
\hline Pendiente (PEN) & & & & & & & & - & $\begin{array}{l}r_{s}=-0,025 \\
P=0,946\end{array}$ \\
\hline Altitud (ALT) & & & & & & & & & - \\
\hline
\end{tabular}

significativa con la densidad total de árboles (prueba de Spearman, $\mathrm{r}_{\mathrm{s}}=0,845 ; \mathrm{P}=0,002$ ) (Tabla 4). Densidad de plántulas de Legrandia se asocia positiva y significativamente con la densidad de árboles de Legrandia (prueba de Spearman, $\left.\mathrm{r}_{\mathrm{s}}=0,838 ; \mathrm{P}=0,002\right)$, área basal total (prueba de Spearman, $\mathrm{r}_{\mathrm{s}}=0,784 ; \mathrm{P}=$ $0,007)$ y área basal de Legrandia (prueba de Spearman, $\mathrm{r}_{\mathrm{s}}=0,729 ; \mathrm{P}=0,017$ ) (Tabla 4). El área basal de Legrandia también presentó una fuerte correlación con la densidad de árboles de Legrandia (prueba de Spearman, $\mathrm{r}_{\mathrm{s}}=0,875 ; \mathrm{P}=$ $0,001)$ y área basal total (prueba de Spearman, $\left.\mathrm{r}_{\mathrm{s}}=0,733 ; \mathrm{P}=0,016\right)$ (Tabla 4). De las variables de sitio, solo el área basal total muestra una correlación positiva y significativa con la altitud (prueba de Spearman, $\mathrm{r}_{\mathrm{s}}=0,709$; $\mathrm{P}=0$,022).

Los resultados del PCA mostraron que el primer componente explica la mayor parte de la variación de los datos (56 \%) (Fig. 5). En el primer componente también se observa que los valores de carga para las variables varían entre 0,5 y 0,8 lo cual sugiere que las variables se encuentran fuertemente autocorrelacionadas
(Fig. 5). Del PCA también es posible inferir que las variables que más contribuyen a explicar la variación de los datos en el primer componente son la densidad total de árboles y el área basal total, ambas con valores de carga superior a 0,8 (Fig. 5). En tanto, para el segundo componente las variables más importantes son la densidad total de brinzales y de Legrandia (Fig. 5).

Relaciones entre variables de estructura de la vegetación e índices de paisaje

Las variables de estructura de la vegetación más representativas (densidad total de árboles y área basal total) se asocian positiva y significativamente con los tres índices de paisaje seleccionados (área, IPM y DBF) (Tabla 5). Otros resultados relevantes muestran que la densidad de árboles de Legrandia presenta una relación positiva y significativa con el área del fragmento y DBF (prueba de Spearman, $r_{s}=$ 0,627; $\mathrm{P}=0,050$ ) (Tabla 5). Área basal de Legrandia está relacionada en forma positiva y significativa con área del fragmento (prueba de 
Spearman, $\left.\mathrm{r}_{\mathrm{s}}=0,628 ; \mathrm{P}=0,050\right)$ y $\mathrm{DBF}$ (prueba de Spearman, $\mathrm{r}_{\mathrm{s}}=0,770 ; \mathrm{P}=0,009$ ) (Tabla 5). $\mathrm{Si}$ bien las relaciones entre densidad total de árboles y plántulas con área del fragmento no son significativas, estas relaciones explican más del $50 \%$ de la variación, al igual que la relación entre densidad de árboles de Legrandia e IPM (Tabla 5).

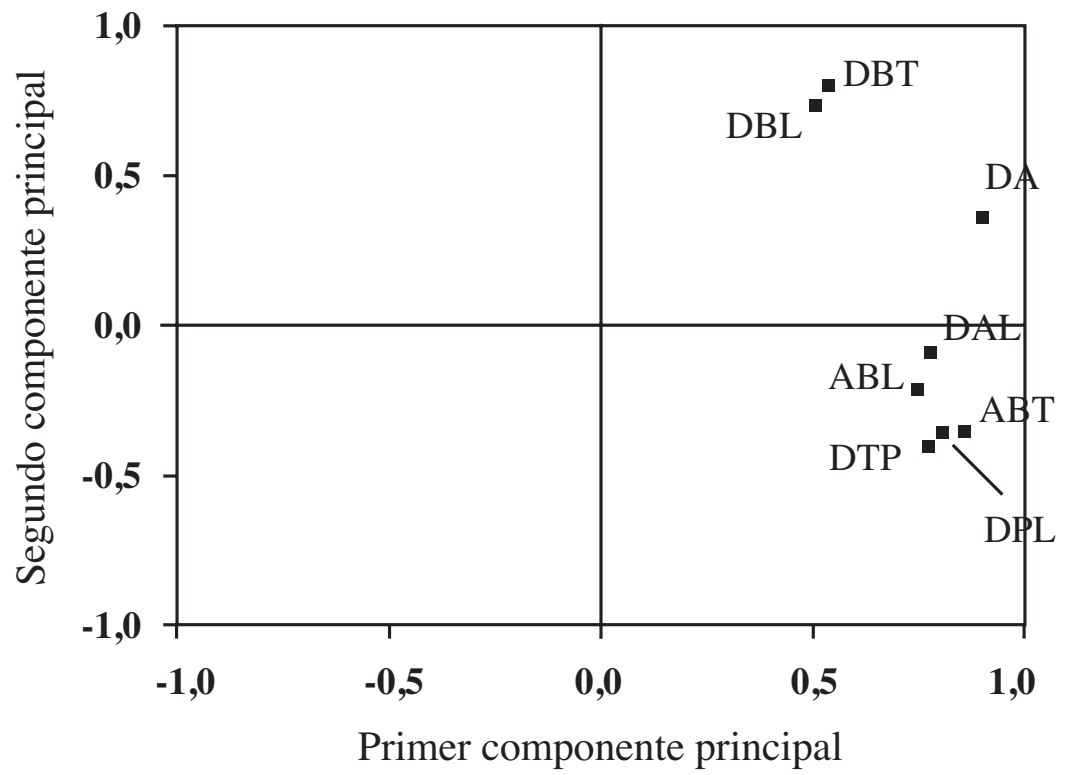

Fig. 5: Gráfico de los dos primeros componentes principales (ambos explican $79 \%$ de la variación total) para variables de estructura de la vegetación de poblaciones de Legrandia medidas en terreno $(\mathrm{n}=10)$. Códigos de variables: $\mathrm{DA}=$ densidad total árboles, $\mathrm{DAL}=$ densidad árboles Legrandia, DTB $=$ densidad total brinzales, DBL $=$ densidad brinzales Legrandia, DTP $=$ densidad total plántulas, $\mathrm{DPL}=$ densidad plántulas Legrandia, $\mathrm{ABT}$ = área basal total, $\mathrm{ABL}=$ área basal Legrandia .

Plot of the first two principal components (accounting for $79 \%$ of total variation) for vegetation structure variables of Legrandia populations measured in the field. Variable codes: DA = total tree density, DAL $=$ tree density of Legrandia, DTB $=$ total sapling density, DBL $=$ sapling density of Legrandia, DTP $=$ overall seedlings density, DPL $=$ seedling density of Legrandia, $\mathrm{ABT}=$ total basal area, $\mathrm{ABL}=$ basal area of Legrandia .

\section{TABLA 5}

Correlación entre índices de paisaje y variables de estructura de la vegetación en poblaciones de Legrandia (prueba de Spearman, $\mathrm{n}=10$ ). Correlaciones estadísticamente significativas indicadas en "negritas"

Correlation between landscape indexes and vegetation structure variables in Legrandia populations (Spearman's test, $\mathrm{n}=$ 10). Statistically significant correlations are indicated in bold typing

\begin{tabular}{|c|c|c|c|}
\hline Variable & Área & $\begin{array}{c}\text { Índice de proximidad } \\
\text { media (IPM) }\end{array}$ & $\begin{array}{l}\text { Distancia al borde } \\
\text { fragmento (DBF) }\end{array}$ \\
\hline Densidad total árboles (DA) & $\mathrm{r}_{\mathrm{s}}=0,537 \mathrm{P}=0,110$ & $r_{s}=0,432 P=0,213$ & $r_{s}=0,673 P=0,033$ \\
\hline Densidad árboles Legrandia (DAL) & $r_{s}=0,627 P=0,050$ & $r_{s}=0,543 P=0,105$ & $r_{s}=0,772 P=0,009$ \\
\hline Densidad total brinzales (DTB) & $r_{s}=0,439 P=0,204$ & $r_{s}=0,401 P=0,250$ & $r_{s}=0,612 P=0,060$ \\
\hline Densidad brinzales Legrandia (DBL) & $r_{s}=0,061 P=0,867$ & $\mathrm{r}_{\mathrm{s}}=0,024 \mathrm{P}=0,947$ & $r_{s}=0,103 \mathrm{P}=0,777$ \\
\hline Densidad total plántulas (DTP) & $r_{s}=0,555 P=0,096$ & $r_{s}=0,462 P=0,179$ & $r_{s}=0,442 P=0,200$ \\
\hline Densidad plántulas Legrandia (DPL) & $r_{s}=0,388 P=0,267$ & $\mathrm{r}_{\mathrm{s}}=0,305 \mathrm{P}=0,392$ & $r_{s}=0,432 P=0,213$ \\
\hline Área basal total (ABT) & $r_{s}=0,689 P=0,028$ & $r_{s}=0,632 P=0,046$ & $r_{s}=0,782 P=0,008$ \\
\hline Área basal Legrandia (ABL) & $r_{s}=0,628 P=0,050$ & $r_{s}=0,468 P=0,172$ & $r_{s}=0,770 P=0,009$ \\
\hline
\end{tabular}




\section{DISCUSIÓN}

\section{Fragmentación del paisaje}

El área estudiada se encuentra dominada por fragmentos de bosque nativo de muy bajo tamaño (más del $60 \%$ de tamaño inferior a 1 ha), lo cual representa uno de los principales argumentos para afirmar que estos bosques se encuentran sometidos a un fuerte proceso de fragmentación. Los altos niveles de aislamiento y formas más regulares de los fragmentos del área estudiada son coincidentes con el grado de fragmentación señalado por otros estudios de Chile (Bustamante \& Castor 1998, Echeverría et al 2006). Por otra parte, zonas boreales (Fitzsimmons 2003) y tropicales (Cayuela et al. 2006) han reportado menores niveles de fragmentación en términos de fragmentos de menor tamaño. Bustamante \& Castor (1998) encontraron patrones de distribución similar en cuanto a tamaño (más del $60 \%$ de fragmentos con tamaño inferior a 1 ha) de fragmentos con dominancia de ruil en la zona costera del área de estudio. Otros estudios llevados a cabo en la zona costera del área de estudio (Lara et al. 1989, Lara et al. 1996, Echeverría et al. 2006) han reportado altas tasas de deforestación y fragmentación, lo cual ha traído como consecuencia la conformación de un paisaje en el cual el bosque original ha sido removido casi completamente por plantaciones de especies exóticas (principalmente de Pinus radiata) y los fragmentos remanentes se encuentran inmersos en esta matriz. La deforestación en el área de estudio fue evaluada entre los años 1987 y 1996 mediante la superposición de mapas temáticos, destacando que en dicho período hubo un cambio significativo y cuya causa principal era la sustitución de bosque nativo por plantaciones de Pinus radiata (Olivares 1999).

No obstante, debido a que el área de estudio es menos accesible por sus condiciones topográficas y menor densidad de caminos, en comparación a la zona costera, es posible encontrar lugares con presencia de grandes fragmentos dominando el paisaje y una acotada superficie de bosques de especies exóticas que no supera el $10 \%$. La materialización de nuevos proyectos en la zona y el crecimiento que ha experimentado la industria forestal en los últimos años (Lara et al. 2003) hace suponer que la demanda por sitios para el establecimiento de nuevas plantaciones de especies exóticas seguirá en aumento, lo cual promoverá la fragmentación de los bosques, tal como ha ocurrido en la zona costera de esta región (Echeverría el al. 2006). Esta situación señala la necesidad de proteger estos ecosistemas remanentes para que el proceso no continúe ocurriendo (Lara et al. 2003).

Patrón de ocupación de los fragmentos con presencia de Legrandia

Los resultados respecto al patrón de ocupación sugieren que las poblaciones de Legrandia tienden a ocupar fragmentos de menor tamaño y mayor aislamiento. Como muestran los resultados, en el paisaje dominan este tipo de características (bajo tamaño de fragmentos y en condiciones de aislamiento) y probablemente las poblaciones están restringidas a las condiciones predominantes. Esto último también puede apoyarse debido a que la distribución de frecuencias de los fragmentos con presencia de Legrandia es similar a los fragmentos de bosques remanentes que dominan en el área, basado en que no hubo diferencias significativas entre ellas en cuanto a tamaño y aislamiento (Fig. 4). La solidez de estos resultados podrían ser objetables, debido al escaso número de fragmentos (Virgós \& García 2002) donde está presente la especie, aspecto que debiese ser explorado en futuras investigaciones con otras especies amenazadas.

Aunque no es posible inferir cuál era la estructura espacial de los fragmentos con presencia de Legrandia en el pasado, se estima que el proceso de deforestación que opera en el área de estudio, el cual alcanza aproximadamente a $2,5 \%$ en los últimos 12 años (Altamirano et al. datos no publicados), haya causado una progresiva fragmentación y por consiguiente modificación del hábitat de la especie. Es así como los altos niveles de deforestación han provocado una rápida fragmentación del bosque nativo en la zona costera del área de estudio modificando progresivamente la estructura de los bosques nativos desde hace 30 años (Echeverría et al. 2006). Como se ha señalado Legrandia presenta una distribución bastante restringida y con hábitats específicos (Hechenleitner et al. 2005, Martínez et al. en prensa), pero probablemente hace 30 años la mayor parte de estas poblaciones 
se pueden haber encontrado en fragmentos de mayor tamaño y sin restricciones de aislamiento, lo cual pudo haberse modificado por el actual proceso de deforestación y fragmentación que opera en esa parte del país.

\section{Impactos de la fragmentación en la estructura de la vegetación}

Los resultados que se infieren de las relaciones entre las variables de estructura de la vegetación y los índices de paisaje, sugieren que sería esperable una mayor área basal y un mayor número de árboles, brinzales y plántulas de Legrandia en fragmentos de mayor tamaño, más cercanos unos a otros y ubicados a mayor distancia del borde del fragmento (Tabla 5). Efectos similares del tamaño de los fragmentos sobre la densidad de juveniles han sido reportados en poblaciones de Dombeya acutangula en el Archipiélago Macarene, océano Índico (Gigord et al. 1999). La fragmentación, medida en términos de tamaño de fragmentos, también ha producido efectos significativos sobre la calidad de las semillas de Lapageria rosea en bosques fragmentados de la zona central de Chile, lo cual se señala puede inducir negativamente la sobrevivencia de las poblaciones a largo plazo (Henríquez 2004).

El aislamiento de los fragmentos también ha sido determinante en la presencia de algunas especies, como es señalado en algunos ecosistemas de Europa occidental (Piessens et al. 2005). Esta condición de aislamiento también puede afectar indirectamente la cantidad y calidad de las interacciones biológicas, como la dispersión de semillas, debido a la reducción y eficiencia de los agentes polinizadores (Bustamante \& Grez 1995). En el caso de Legrandia, la dispersión de las semillas podría verse limitada aún más al presentar un fruto de gran tamaño (Hechenleitner et al. 2005).

Lindenmayer \& Franklin (2002) señalan que condiciones ambientales como luminosidad, temperatura y humedad se ven claramente afectadas bajo una determinada distancia al borde y forma de un fragmento, lo cual puede afectar la presencia de una especie. Los cambios ecológicos producidos por el efecto borde dependen de la variable de interés, lugar y especie bajo análisis, existiendo ejemplos que varían desde 15 a más de $300 \mathrm{~m}$ de distancia de penetración del efecto borde (Mendoza et al.
2005). Cambios microclimáticos como temperatura y humedad pueden verse afectados en un rango de 15 a $50 \mathrm{~m}$, distancia que podría considerarse crítica para que se produzcan efectos en estas poblaciones (Jose et al. 1996, Mendoza et al. 2005). En el presente estudio se observó que la distancia al borde del fragmento condiciona fuertemente la presencia de Legrandia, lo cual podría explicarse porque la especie requiere de hábitats sombríos y con mayor humedad (Serra et al. 1986), condiciones que se ven afectadas por la distancia al borde del fragmento.

Efectos de la fragmentación no medidos en estudio, como la pérdida de variabilidad genética merecen ser atendidos, dado que se han reportado consecuencias negativas sobre algunas especies, como es el caso Trillium camschatcense, una hierba que domina en bosques de Japón (Tomimatsu \& Ohara 2003). En el caso de Legrandia, algunos resultados muestran altos niveles de endogamia en algunas poblaciones localizadas en el área de estudio como Bullileo, La Balsa, Minas del Prado y San Fabián 4 (Martínez et al. datos no publicados). En caso de persistir la fragmentación de las poblaciones estudiadas este efecto podría ser relevante para la sobrevivencia de las poblaciones.

\section{Medidas de conservación}

Debido a la condición actual de especie en categoría vulnerable, y al ser una especie endémica y monotípica, no parece lógica la ausencia de estas poblaciones en el Sistema Nacional de Áreas Protegidas del Estado (SNASPE), sistema que no ha considerado criterios como la riqueza de especies y endemismos en la protección de ecosistemas (Armesto et al. 1998). Siguiendo las recomendaciones de Gigord et al. (1999) un primer avance sería determinar cuáles poblaciones se encuentran más amenazadas y sobre estas ejercer las acciones más urgentes de conservación, además de proteger aquellas que presenten mayor integridad. En este caso se recomienda ejercer las acciones de conservación en aquellas poblaciones que presentaron menor presencia (<200 árboles/ha) de la especie como Radal, Coihueco 18, Coihueco 3 y Coihueco 9 (Tabla 2). Posterior a esto, se hace necesaria la creación de nuevas áreas protegidas públicas o 
privadas para resguardar las poblaciones con mayor riesgo y los fragmentos en mejor estado, y como medida alternativa promover su conservación ex situ a través de convenios de plantación, en cooperación con propietarios privados en fragmentos sin presencia de Legrandia, dentro del rango de distribución de la especie. Al existir un número muy reducido de poblaciones también se considera importante promover programas de conservación in vitro, para producción de plantas en viveros, existiendo algunos avances en este sentido, como los descritos por Uribe-Moraga \& Cifuentes (2004) para esta misma especie.

Debido al aislamiento de las poblaciones sería adecuado aumentar la conectividad entre ellas, ya que esto puede aumentar las posibilidades de sobrevivencia de la especie en el tiempo (Piessens et al. 2005).

En áreas sometidas a procesos de fragmentación y con presencia de especies invasoras y especies con problemas de conservación, algunos autores recomiendan controlar el avance de las primeras e inducir la polinización en las nativas (Gigord et al. 1999). Ambas medidas pueden evaluarse para las poblaciones de Legrandia que involucró este estudio, debido a la alta presencia de plantaciones de especie exóticas, principalmente de Pinus radiata. Todas estas acciones deben ir acompañadas de un programa de monitoreo que permita evaluar los cambios en el tiempo a escala de paisaje y rodal (Trani \& Giles 1999, Egbert et al. 2002, Lausch \& Herzog 2002).

Los resultados de este estudio muestran que cambios en la estructura del paisaje, asociados al proceso de fragmentación, están relacionados de manera negativa con la presencia de poblaciones de Legrandia, y por esta razón muestran la necesidad urgente de tomar medidas de conservación en estas poblaciones. El estudio sienta un precedente respecto a la vinculación entre información a escala de paisaje e información de estructura de la vegetación medida en terreno, para evaluar el estado de conservación de poblaciones de especies amenazadas que cubren extensiones restringidas de territorio. También, muestra la aplicación de imágenes satelitales para generar mapas de coberturas del suelo y así cuantificar la estructura del paisaje como una forma de medir los niveles de fragmentación. Esta herramienta alcanza un mayor potencial cuando se realizan estudio temporales usando imágenes satelitales de diferentes fechas (Staus et al. 2002, Cayuela et al. 2006, Echeverría et al. 2006). Esta innovación metodológica ofrece la posibilidad de monitorear los cambios producidos en el tiempo a fin de evaluar la efectividad de determinadas estrategias de conservación para estas poblaciones.

\section{AGRADECIMIENTOS}

Esta investigación es parte de las actividades del autor principal en el Programa Integrado de Doctorado en Ciencias Forestales de la Universidad Austral de Chile - Universidad de Concepción. Se agradece el financiamiento de los estudios de doctorado de A. Altamirano al proyecto MECESUP AUS 0103. Este trabajo contó con el apoyo financiero del proyecto BIOCORES (Biodiversity conservation, restoration and sustainable use in fragmented forest landscapes) contrato ICA-CT-200110095 financiado por la Comunidad Europea, y el Núcleo Científico FORECOS P04-065-F (Servicios ecosistémicos del bosque nativo a sistemas acuáticos bajo fluctuaciones climáticas) financiado por la iniciativa Científica Milenio de MIDEPLAN. Por facilitar datos de localización de poblaciones de Legrandia agradecemos al proyecto "Programa de conservación integrado para las especies forestales endémicas amenazadas de Chile" $\mathrm{N}^{\circ}$ 162/11/012, financiado por Darwin Initiative del Departamento de Medio Ambiente del Reino Unido. Finalmente, agradecemos el aporte en el trabajo a las siguientes personas: Víctor Sandoval, Sandra Molina, Patricio Romero, Camila Martínez, Carlos Le Quesne, Carlos Zamorano, Jaime Cuevas y Oscar Thiers. También por el apoyo en terreno se agradece a Ricardo González, Fernando Bustos y Felipe Osorio.

\section{LITERATURA CITADA}

ARMENTERAS D, F GAST \& H VILLAREAL (2003) Andean forest fragmentation and the representativeness of protected natural areas in the eastern Andes, Colombia. Biological Conservation 113: 245-256

ARMESTO J, R ROZZI, C SMITH-RAMÍREZ \& MT ARROYO (1998) Conservation targets in South American temperate forests. Science 282: 1271-1272. 
BANKS S C, GR FINLAYSON, SJ LAWSON, DB LINDENMAYER, D PAETKAU, SJ WARD \& AC TAYLOR (2005) The effects of habitat fragmentation due to forestry plantation establishment on the demography and genetic variation of a marsupial carnivore, Antechinus agilis. Biological Conservation 122: 581-597.

BENOIT I (1989) Libro rojo de la flora terrestre de Chile. Corporación Nacional Forestal, Santiago, Chile. $157 \mathrm{pp}$.

BUSTAMANTE R \& A GREZ (1995) Consecuencias ecológicas de la fragmentación de los bosques nativos. Ambiente y Desarrollo (Chile) 11: 58-63.

BUSTAMANTE RO \& C CASTOR (1998) The decline of an endangered temperate ecosystem: the ruil (Nothofagus alessandrii) forest in central Chile. Biodiversity and Conservation 7: 1607-1626.

CAYUELA L, JM REY BENAYAS \& C ECHEVERRÍA (2006) Clearance and fragmentation of tropical montane forests in the Highlands of Chiapas, Mexico (1975-2000). Forest Ecology and Management 226: 208-218

CONAF, CONAMA, BIRF, UNIVERSIDAD AUSTRAL DE CHILE, PONTIFICIA UNIVERSIDAD CATÓLICA DE CHILE \& UNIVERSIDAD CATÓliCA DE TEMUCO (1999) Catastro y evaluación de los recursos vegetacionales nativos de Chile. Informe Nacional con Variables Ambientales, Santiago, Chile. 88 pp

DALE VH \& SM PEARSON (1997) Quantifying habitat fragmentation due to land-use change in Amazonia. En: Laurance WF \& RO Bierregaard (eds) Tropical forest remnants: ecology, management and conservation of fragmented communities: 400-410. University of Chicago Press, Chicago, Illinois, USA.

DINERSTEIN E, DM OLSON, DJ GRAHAM, AL WEBSTER, SA PRIMM \& MP BOOKBINDER (1995) Una evaluación del estado de conservación de las ecorregiones terrestres de América Latina y el Caribe. Banco Mundial/WWF, Washington, District of Columbia, USA. 129 pp.

DYTHAM C (2003) Choosing and using statistics. A biologist's guide. Second edition. Blackwell Publishing. Oxford, United Kingdom. xiii +248 pp.

ECHEVERRÍA C, D COOMES, J SALAS, JM REYBENAYAS, A LARA \& A NEWTON (2006) Rapid deforestation and fragmentation of Chilean temperate forests. Biological Conservation 130: 481-494.

EGBERT S L, S PARK, KP PRICE, R LEE, J WUC \& MD NELLIS (2002) Using conservation reserve program maps derived from satellite imagery to characterize landscape structure. Computers and Electronics in Agriculture 37: 141-156.

ERDAS (1999) Erdas field guide. Fifth edition, revised and expanded. Erdas Inc., Atlanta Georgia, USA. $698 \mathrm{pp}$

ESTADES CF \& SA TEMPLE (1999) Deciduous-forest bird communities in a fragmented landscape dominated by exotic pine plantations. Ecological Applications 9: 573-585.

FERRERAS P (2001) Landscape structure and asymmetrical inter-patch connectivity in a metapopulation of the endangered Iberian Lynx. Biological Conservation 100: 125-136.

FITZSIMMONS M (2003) Effects of deforestation and reforestation on landscape spatial structure in boreal Saskatchewan, Canada. Forest Ecology and Management 174: 577-592.
FRANKLIN S (2001) Remote sensing for sustainable forest management. Lewis Publishers, Boca Raton, Florida, USA. 407 pp.

GIGORD L, F PICOT \& J SHYCOFF (1999) Effects of habitat fragmentation on Dombeya acutangula (Sterculiaceae), a native tree on La Réunion (Indian Ocean). Biological Conservation 88: 43-51.

GREZ A, R BUSTAMANTE, J SIMONETTI \& L FAHRIG (1998) Landscape ecology and habitat fragmentation: the case of the ruil forest in Chile. En: Salinas-Chavez E \& J Middleton (eds) Landscape ecology as a tool for sustainable development in Latin America / La ecología del paisaje como base para el desarrollo sustentable en América Latina. Libro electrónico: http:// www.brocku.ca/epi/lebk/lebk.html

GUSTAFSON EJ \& GR PARKER (1992) Relationships between landcover proportion and indices of landscape spatial pattern. Landscape Ecology 7: 101-110.

HECHENLEITENER P, MF GARDNER, PI THOMAS, C ECHEVERRÍA, B ESCOBAR, P BROWNLESS \& C MARTÍNEZ (2005) Plantas amenazadas del centro-sur de Chile. Distribución, conservación y propagación. Primera edición. Trama Impresores S.A., Valdivia, Chile. 188 pp.

HENRÍQUEZ CA (2004) Efecto de la fragmentación del hábitat sobre la calidad de las semillas en Lapageria rosea. Revista Chilena de Historia Natural 77: 177-184.

JOSE S, AR GILLESPIE, SJ GEORGE \& BM KUMAR (1996) Vegetation responses along edge-to-interior gradients in a high altitude tropical forest in peninsular India. Forest Ecology and Management 87: 5 1-62.

KERR J \& M OSTROVSKY (2003) From space to species: ecological applications for remote sensing. Trends in Ecology and Evolution 18: 299-305.

LANDRUM LR (1986) A Monograph of the Genus Campomanesia, Pimenta, Blepharocalyx, Legrandia, Acca, Myrrhinium, and Luma (Myrtaceae). Flora Neotrópica 45: 1-173.

LARA A, D SOTO, J ARMESTO, P DONOSO, C WERNLI, L NAHUELHUAL \& F SQUEO (eds) (2003) Componentes científicos clave para una política nacional sobre usos, servicios y conservación de los bosques nativos chilenos. Universidad Austral de Chile, Iniciativa Científica Milenio de Mideplan, Gobierno de Chile. 134 pp.

LARA A, C DONOSO \& JC ARAVENA (1996) La conservación del bosque nativo en Chile: problemas y desafíos. En: Armesto J, C Villagrán \& M Arroyo (eds) Ecología de los bosques nativos de Chile: 335-362. Editorial Universitaria, Santiago, Chile.

LARA A, JC ARAVENA, R VILLALBA, A WOLODARSKY-FRANKE, B LUCKMAN \& R WILSON (2001) Dendroclimatology of highelevation Nothofagus pumilio forests at their northern distribution limit in the central Andes of Chile. Canadian Journal of Forest Research 31: 925-936.

LARA A, L ARAYA, J CAPELLA, M FIERRO \& A CAVIERES (1989) Evaluación de la destrucción y disponibilidad de los recursos forestales nativos en la VII y VIII Región. Informe Técnico, Comité Prodefensa Fauna y Flora, Santiago, Chile. 22 pp.

LAUSCH A \& F HERTZOG (2002) Applicability of landscape metrics for the monitoring of landscape change: issues of scale, resolution and interpretability. Ecological Indicators 2: 3-15. 
LI X, HS HE, X WANG, R BU, Y HU \& Y CHANG (2004) Evaluating the effectiveness of neutral landscape models to represent a real landscape. Landscape and Urban Planning 69: 137-148.

LILLESAND TM \& RW KIEFER (1994) Remote Sensing and Image Interpretation. John Wiley and Sons, New York, New York, USA. 750 pp.

LINDENMAYER DB \& JF FRANKLIN (2002) Conserving forest biodiversity. A comprehensive multiscaled approach. Island Press, Washington, District of Columbia, USA. $351 \mathrm{pp}$.

MCGARIGAL K \& BJ MARKS (1995) FRAGSTATS: spatial pattern analysis program for quantifying landscape structure. United States Department of Agriculture, Forest Service, General Technical Report PNW-351.

MARTÍNEZ C, P HECHENLEITNER, MF GARDNER, C DONOSO \& B ESCOBAR (en prensa) Legrandia concinna (Phil.) Kausel. Luma, luma del norte, luma blanca. Familia: Myrtaceae. En: Donoso C (ed) Autoecología de las especies arbóreas de los bosques templados de Chile y Argentina. Trama Impresores, Valdivia, Chile.

MATHER P (1999) Computer processing of remotelysensed images. An introduction. Second edition. John Wiley \& Sons, Chichester, New York, USA. $292 \mathrm{pp}$.

MENDOZA E, J FAY \& R DIRZO (2005) A quantitative analysis of forest fragmentation in Los Tuxtlas, southeast Mexico: patterns and implications for conservation. Revista Chilena de Historia Natural 78: 451-467.

NOSS R (2000) High-risk ecosystems as foci for considering biodiversity and ecological integrity in ecological risk assessments. Environmental Science \& Policy. 3: 321-332.

OLIVARES P (1999) Sustitución de bosque nativo por otros usos del suelo en dos sectores de la precordillera andina de la VII Región, entre los años 1987 y 1996. Tesis de grado Ingeniería Forestal, Facultad de Ciencias Forestales, Universidad Austral de Chile, Valdivia, Chile. 81 pp.

PEZOA LS (2003) Recopilación y análisis de la variación de las temperaturas (período 1965-2001) y las precipitaciones (período 1931-2001) a partir de la información de estaciones meteorológicas de Chile entre $\operatorname{los} 33^{\circ}$ y $53^{\circ}$ de latitud sur. Tesis de grado Ingeniería Forestal, Facultad de Ciencias Forestales, Universidad Austral de Chile, Valdivia, Chile. 99 pp.

PIESSENS K, O HONNAY \& M HERMY (2005) The role of fragment area and isolation in the conservation of heathland species. Biological Conservation 122: 61-69.

RODRÍGUEZ R, M QUEZADA \& O MATTHEI (1983) Flora arbórea de Chile. Ediciones Universidad de Concepción, Concepción, Chile. 403 pp.
SAUNDERS D, RJ HOBBS, CR MARGULES (1991) Biological consequences of ecosystem fragmentation: a review. Conservation Biology 5: $18-32$.

STAUS N, J STRITTHOLT, D DELLASALA \& R ROBINSON (2002) Rate and patterns of forest disturbance in the Klamath-Siskiyou ecoregion, USA between 1972 and 1992. Landscape Ecology 17: 455-470.

SERRA MT, R GAJARDO \& A CABELLO (1986) Programa de protección y recuperación de la flora nativa de Chile. Ficha técnica de especies amenazadas; Legrandia concinna Koch. (Myrtaceae), especie vulnerable. Universidad de Chile, Santiago, Chile. 21 pp.

SKOLE D \& C TUKER (1993) Tropical deforestation and habitat fragmentation in the Amazon: satellite data from 1978 to 1988. Science 260: 1905-1910.

TOMIMATSU H \& M OHARA (2003) Genetic diversity and local population structure of fragmented populations of Trillium camschatcense (Trilliaceae). Biological Conservation 109: 249258.

TRANI MK \& RH GILES (1999) An analysis of deforestation: metrics used to describe pattern change. Forest Ecology and Management 114: 459470 .

TURNER M, R GARDNER \& R O' NEILL (2001) Landscape ecology in theory and practice. Patterns and process. Springer-Verlag, New York, New York, USA. $401 \mathrm{pp}$

URIBE-MORAGA M \& L CIFUENTES (2004) Aplicación de técnicas de cultivo in vitro en la propagación de Legrandia concinna. Bosque (Chile) 25: 129-135.

VENEMA HD, PH CALAMAI \& P FIEGUTH (2005) Forest structure optimization using evolutionary programming and landscape ecology metrics. European Journal of Operational Research 164: 423-439.

VERBOOM B \& R VAN APELDOORN (1990) Effects of habitat fragmentation on the red squirrel Sciurus vulgaris. Landscape Ecology 4: 171-176.

VERGARA PM \& JA SIMONETTI (2004) Avian responses to fragmentation of the Maulino forest in central Chile. Oryx 38: 383-388.

VIRGÓS E \& F GARCÍA (2002) Patch occupancy by stone martens Martes foina in fragmented landscapes of central Spain: the role of fragment size, isolation and habitat structure. Acta Oecologica 23: 231-237.

WALTER KS \& HJ GILLET (1998) 1997 IUCN Red list of threatened plants. Compiled by the World Conservation Monitoring Centre. International Union for Conservation of Nature, Gland, Switzerland. $862 \mathrm{pp}$

WILLSON MF, TI DESANTO, C SABAG \& JJ ARMESTO (1994) Avian communities of fragmented south-temperate rain-forests in Chile. Conservation Biology 8: 508-520. 